\title{
An Open Data Set for Rail Vehicle Positioning Experiments*
}

\author{
Michael Roth ${ }^{1}$ and Hanno Winter ${ }^{2}$
}

\begin{abstract}
This paper describes an openly available data set for rail vehicle positioning experiments. The data were collected using the DLR research vehicle RailDriVE on a segment of the harbor railway of Braunschweig, Germany, in February 2019. Several sensors of the RailDriVE equipment and an additional self-sufficient system provided by Technische Universität Darmstadt were employed, including two GNSS receivers, two inertial measurement units (IMU), and several speed and distance sensors (radar, optical, odometer) from the rail domain. Front-facing camera data has been included for documentation purposes. In order to simplify its use, some pre-processing steps were applied to the data, mainly to have common time and coordinate frames. Furthermore, example and reference positioning solutions have been included. The data set is described in detail, with information about the individual sensors and the data set structure (with parameters, raw, pre-processed, and reference data). Our work should be seen as a step towards more open and data-driven research in the rail domain, where experiments are difficult and costly. It is our hope to provide a solid basis for many different research efforts that provide the required technological advances for the rail sector.
\end{abstract}

\section{INTRODUCTION}

Accurate and reliable rail vehicle positioning is a crucial requirement for future railway systems. Given the position of all trains, their distances can be decreased in order to increase the capacity of the railway network. Fully or partially automated trains require position and velocity information for safety and control purposes. Position information of a fleet of shunters can be used for optimized resource allocation and simplified management. From an economic perspective, a focus on on-board technology is important because of the high costs of infrastructure-side positioning solutions. Accordingly, the recent years have seen much research on the topic [1].

In contrast to research on, e. g., pedestrian or road vehicle positioning, experimental data from the rail domain is harder to obtain for several reasons. The railway sector is less agile than the automotive sector due to the high costs and long lifetimes of the vehicles and the infrastructure. Inservice vehicles from the 1960 s are a common sight in many shunting yards. Strict safety regulations and concerns about interference with existing systems further complicate

*This work has received funding from the Shift2Rail Joint Undertaking (JU) under grant agreement No 826206. The JU receives support from the European Union's Horizon 2020 research and innovation programme and the Shift2Rail JU members other than the Union.

${ }^{1}$ Institute of Transportation Systems, German Aerospace Center (DLR), Braunschweig, Germany m. rothedlr.de

${ }^{2}$ Control Methods and Robotics Laboratory, Technische Universität Darmstadt, Germany hanno.winterermr.tu-darmstadt.de

1,2 Both authors contributed equally to this work. the preparation and performance of experiments. Most researchers do not have direct access to a rail vehicle and a track network. The costs of performing experiments with a rented vehicle, driver, and infrastructure are high. All of the above points lead to the fact that little data is openly available and that much of the research is performed on simulated data only. This is in contrast to the automotive domain, where data-driven research dominates the recent literature. Many advances have been facilitated by openly available data sources such as the KITTI data set [2].

This paper addresses this gap and provides an experimental data set that can be openly accessed by researchers and students [3]. The data were jointly collected by the German Aerospace Center (DLR) and Technische Universität Darmstadt (TU Darmstadt) using the DLR research vehicle RailDriVE in February 2019. The employed sensors include different GNSS (global navigation satellite systems) receivers, inertial measurement units (IMU), an odometer, as well as an optical and a radar speed sensor for rail use. The data can be used as a starting point for research work or student theses. Novel and established algorithms for many different sub-problems can be tested on the data, to facilitate their comparison and make results and insights more accessible. Often overlooked practical aspects such as sensor calibration and synchronization can be addressed. It is our belief that a move towards more open research paradigms will help advance new technologies faster and strengthen the overall rail domain. In order to promote this idea the authors participate in the LRT (localization reference train) initiative, a group of universities and research institutes that jointly work on advancing rail vehicle positioning research [4].

Our paper should be seen as a step towards open data in the rail domain. Because the data were collected on a research vehicle there are obvious limitations, e. g. in terms of speed or driven distance. However, the data realistically represent, e.g., shunting actions. The area in which the tests were performed provides some of the typical challenges, e.g., parallel tracks in close proximity and GNSS obstructions. The collected data can be adjusted to cater many different research challenges (e. g., by removing or altering parts of the GNSS data) while being accurate enough to serve as reference solution. Further experiments are being planned, again with the intention of publishing the data. Interested readers can follow the LRT activities for further information [4].

The outline of the paper is as follows. The test vehicle, infrastructure, and the sensors are described in Sec. II. Details about the data set are described in Sec. III. Prospective research directions using the data are discussed in Sec. IV. Finally, concluding remarks are provided in Sec. V. 


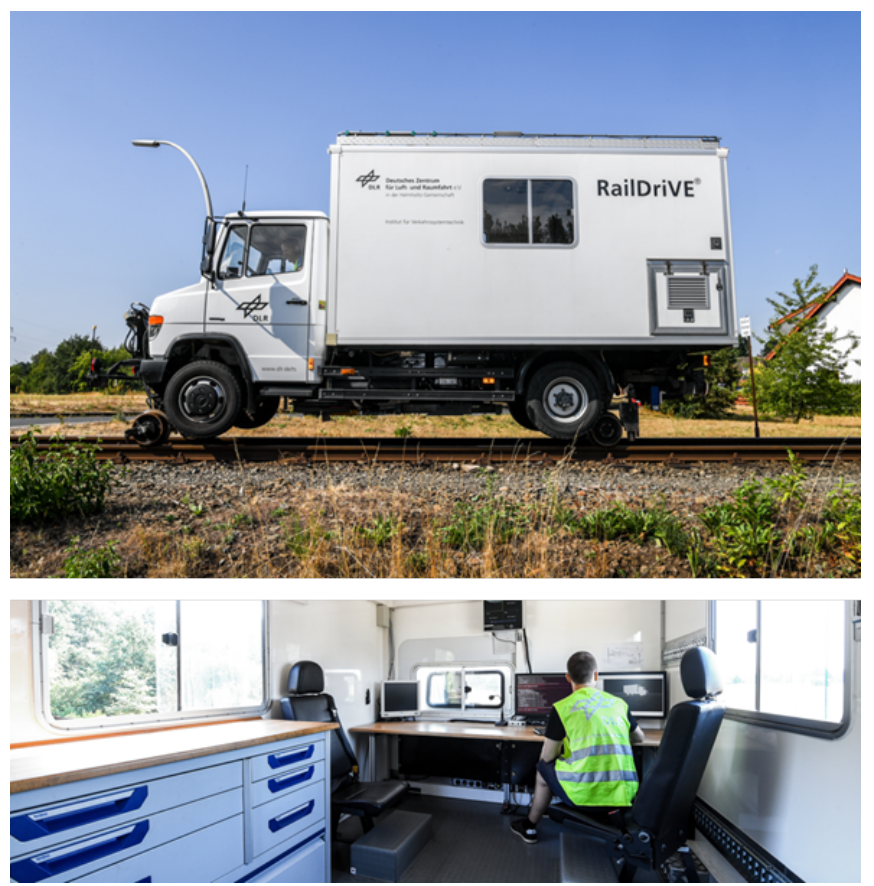

Fig. 1. Views of the test vehicle RailDriVE.

\section{The Vehicle, Sensors, And Test Track}

The following paragraphs present information about the experimental set-up for data collection.

\section{A. The Test Vehicle RailDriVE}

The RailDriVE (Rail Driving Validation Environment) is a mobile research laboratory of the DLR Institute of Transportation Systems. Based on a 2008 Mercedes Benz Vario, the vehicle is equipped with an extra set of rail wheels and hydraulics that facilitate its operation on both road and rails ${ }^{1}$. Since 2008 the RailDriVE has been used for railrelated research on topics such as positioning [5], condition monitoring [6], communication, and mapping [7]. Fig. 1 provides views of the vehicle and the work station in its trunk.

As mobile laboratory, the RailDriVE is equipped with a rich sensor set-up including several high-performance GNSS receivers, several speed sensors from the rail domain, an inertial measurement unit, and two laser scanners. The sensors are connected to a powerful Ubuntu computer that runs Robot Operating System (ROS) [8] to time-stamp and record all data. With individual sensor modules written in $\mathrm{C}++$ and Python, ROS provides a flexible and convenient framework for data acquisition and online processing.

It is obvious that the RailDriVE cannot cater to all relevant positioning scenarios, e.g., because of the small wheel diameters and the limited maximum speed $(50 \mathrm{~km} / \mathrm{h}$, $25 \mathrm{~km} / \mathrm{h}$ on the test infrastructure). It is operated with the permission of the railway infrastructure manager only where it does not interfere with the regular traffic. In contrast to in-service vehicles that underlie strict regulations, however,

${ }^{1}$ Modification by Zweiweg, https://www.zweiweg.de/en/. it is a flexible carrier of technology. Extra sensors or other equipment can be mounted and tested with shorter preparation times. Due to its road and rail use, fast transport at low costs is possible also to remote rail infrastructures. These points make the RailDriVE a very viable asset, e.g., for experiments related to shunting on smaller infrastructures.

\section{B. The RailDriVE Sensors}

The following list contains information about the RailDriVE sensors selected for the data set.

- The GNSS data are provided by a multi-constellation dual frequency Javad Sigma receiver with a roofmounted antenna at a rate of $5 \mathrm{~Hz}$.

- 3D accelerations and turn rates at $100 \mathrm{~Hz}$ are provided by an XSens MTi-G-700 inertial measurement unit.

- A Doppler radar by Siemens mounted under the vehicle provides measurements of the speed, distance, and driving direction at a rate of $5 \mathrm{~Hz}$.

- An optical speed sensor, Correvit Rail, provides measurements of the speed, distance, and driving direction. Information about the measurement principle can be found in [9].

- The front wheels are equipped with a pulse generator that provides measurements of the driven distance, speed, and direction.

- A dash camera was used to record image data $(1024 \times 768$ pixels $)$ with a sampling time of 0.6 seconds.

\section{The TU Darmstadt Sensor System}

The iNat-M200/STN is a MEMS-based inertial navigation system (INS) with an integrated L1/L2 GNSS receiver and tightly coupled INS/GNSS data fusion capabilities. The sensor system is not part of the RailDriVE equipment. It was provided by TU Darmstadt and installed completely selfsufficient, with its own power supply, GNSS antenna, and internal storage. Mounted on a steel plate together with a battery pack it was placed on the floor in the vehicle trunk on a thin rubber mat, as illustrated in Fig. 2. The GNSS antenna was installed on the vehicle roof.

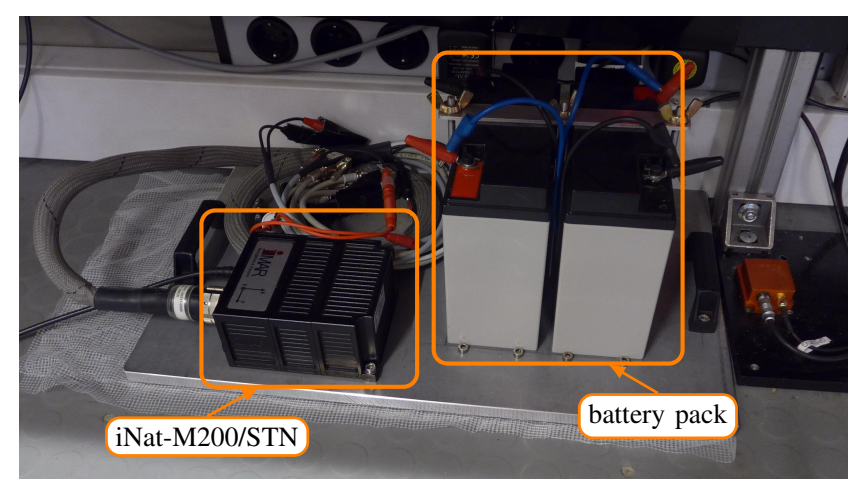

Fig. 2. Additional self-sufficient installation of iNat-M200/STN in the RailDriVE's trunk.

The iNat-M200/STN provides the raw measurement data of the IMU (3D accelerations and turn rates) together with an internally calculated position velocity time (PVT) solution 
of the GNSS receiver. Furthermore, it is possible to store all GNSS observables in a Receiver Independent Exchange Format (RINEX) file for post-processing purposes. Besides these raw data, the sensor also provides an internally calculated positioning solution from a tightly coupled INS/GNSS sensor fusion which may be used as a baseline for other positioning solutions (c. f. Section III-D).

\section{The Test Track}

The experiments were performed in the harbor railway of Braunschweig, Germany, which comprises about $15 \mathrm{~km}$ of tracks with a connection to the national railway network. A maximum speed of $25 \mathrm{~km} / \mathrm{h}$ is permitted on the tracks.

A segment of $1200 \mathrm{~m}$ was repeatedly driven back and forth. The test segment includes parallel tracks in close proximity, several switches, and a bridge above the rails.

\section{The DATA SET}

The data set was recorded on February 22, 2019, and is openly available at [3]. Relevant information about the data is summarized in Fig. 3.

\begin{tabular}{|c|c|}
\hline General: & $\begin{array}{l}\text { - Duration: } 26: 46 \mathrm{~min} \\
\text { - Weather: } \approx 5^{\circ} \mathrm{C}(\mathrm{dry}, \text { cloudy })\end{array}$ \\
\hline Track: & $\begin{array}{l}\text { - Length: } 1.2 \mathrm{~km} \\
\text { - Turnouts (driving forwards): } \\
\text { - trailing }(6 \mathrm{x}) \\
\text { - facing }(3 \mathrm{x}) \\
\text { - GNSS obstacles: } \\
\text { - bridge }(1 \mathrm{x}) \\
\text { - freight trains }(1 \mathrm{x})\end{array}$ \\
\hline Movement: & $\begin{array}{l}\text { - Speed: } \approx 0 \ldots 18 \mathrm{~km} / \mathrm{h} \\
\text { - Repetitions: } \\
\text { - forwards }(3 \mathrm{x}) \\
\text { - backwards }(2 \mathrm{x})\end{array}$ \\
\hline Sensors: & $\begin{array}{l}\text { - } \text { GNSS }(2 \mathrm{x}) \\
\text { - } \text { IMU }(2 \mathrm{x}) \\
\text { - } \text { Speed and distance sensors } \\
\text { (3x; odometer, radar, optical) } \\
\text { - Camera }(2 \mathrm{x})\end{array}$ \\
\hline References: & $\begin{array}{l}\text { - loosely coupled GNSS/IMU fusion } \\
\text { solution (implementation included in } \\
\text { the data set) } \\
\text { - tightly coupled GNSS/IMU fusion } \\
\text { solution (proprietary) } \\
\text { - a map data segment }\end{array}$ \\
\hline
\end{tabular}

Fig. 3. Overview of the specifications of the presented data set.

Fig. 4 shows a view from the RailDriVE cockpit to illustrate the weather conditions and gives an impression of railway infrastructure.

The data set follows the data sharing guidelines of the LRT initiative [10], which are being developed with the authors' participation. The guidelines intend to unify the sharing of measurement and reference data in the context of

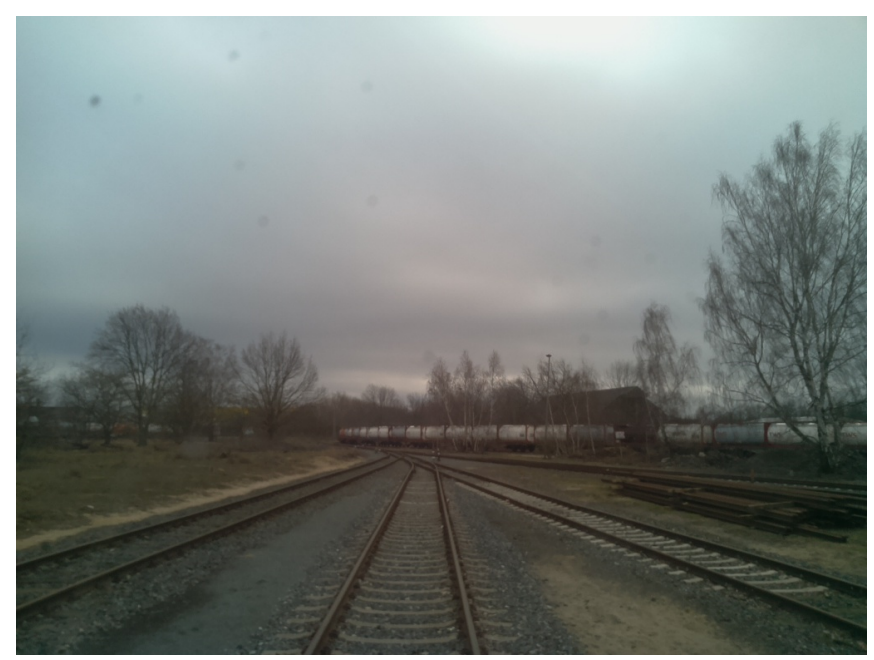

Fig. 4. An image by one of the cameras shows the front view from the vehicle cockpit.

rail vehicle positioning, so as to simplify experiments with data of different sources and to facilitate the comparison of algorithms.

This is the first data set following the LRT guidelines. Hence, its structure and the motivation behind it are briefly introduced next. After that, the sensor and reference data contained in the data set are described, including some hints about its usage.

\section{A. Structure}

As suggested in [10] the data set is structured like depicted in Fig. 5. It is stored in a zip file and the contained data

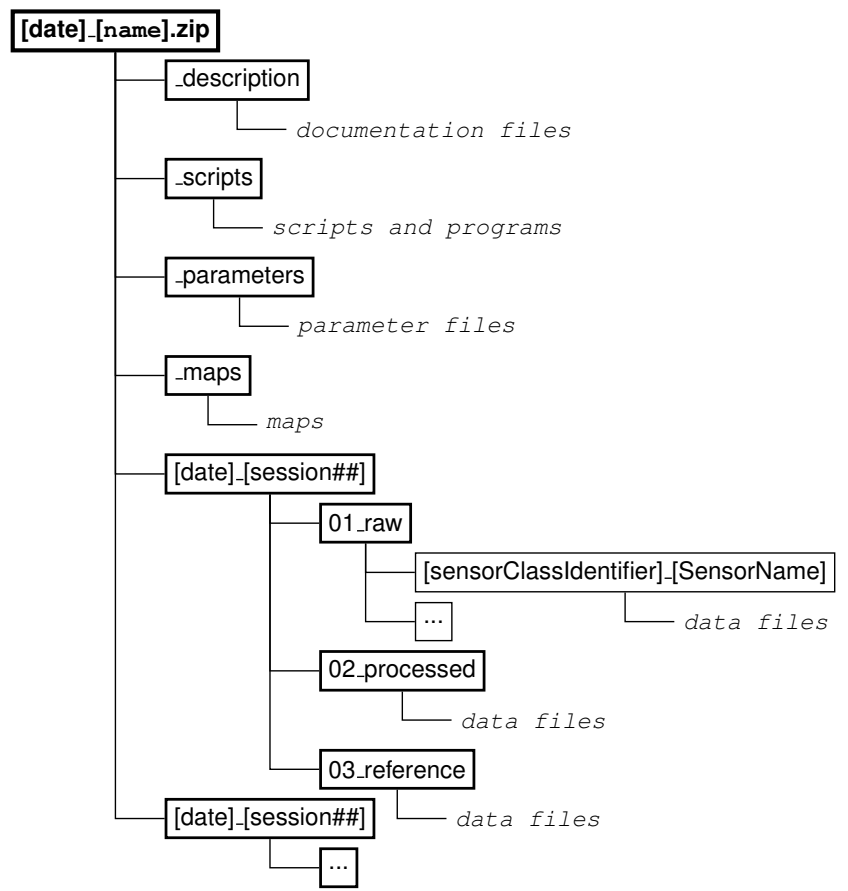

Fig. 5. Structure of the data set (like suggested in [10]). The expressions in square brackets represent placeholders. 
are arranged by sessions. A session is characterized by a continuous stream of recordings. For each session the data is stored in three sub-folders 01_raw, 02_processed and 03_reference.

The raw data folder 01_raw contains all the recorded sensor raw-data without any changes. They are provided mainly for documentation reasons, to make it comprehensible how the processed data in the folder 02_processed have been generated. However, there might be applications where data processing is not applicable and where direct access to the raw data is required.

The data in 02_processed are pre-processed in a unified way. For user convenience, time synchronization, transformation into common reference frames, and the assignment of unified data labels are performed. Especially the latter two processing steps ensure that it becomes possible to easily interchange this data with other data also conforming to [10] without having to change the actual calculations on the data. The reference data folder 03_reference contains data which may be used to evaluate calculation results. Reference data can be, e. g., high precision positioning solutions, sensor fusion results, etc. Possibly available map data are stored in a separate maps folder on the top-level of the data set. As long as it is applicable, the reference data also comply to the reference frames and data labels already used for the processed data. Furthermore, [10] defines common rules for specifying positioning results, to make them better comparable to the results of others, either on the same data or on any other data according to [10].

Further documentation, Matlab scripts for pre-processing and generating the reference data, as well as relevant sensor parameters are provided in respective folders.

\section{B. Raw Data}

This folder contains the raw data of the sensors listed in Section II-B and Section II-C. Additional information about the sensors and their positions on the vehicle can be found directly in the data set [3].

\section{Processed Data}

The processed data provide a convenient starting point for experiments with the data. Before going into the preprocessing details, some data examples are presented. Fig. 6 illustrates the driven path in the network via the Javad GNSS speed and position data for a single journey from start to stop. Hidden by the scatter plots are the tree parallel tracks in the south-east of the infrastructure. A bridge above the rails obstructs the GNSS reception. A closer view including the GNSS data of the entire session is illustrated in Fig. 7. The circles indicate the uncertainty as provided by the receiver. Under the bridge are GNSS outages. In the vicinity of the bridge the measurements are corrupted. Such intermediate GNSS errors can be accounted for in positioning algorithms by conditional selection of GNSS data, gating, or robust estimation schemes.

Example plots of distance and speed measurements are provided in Fig. 8 and Fig. 9, respectively. Fig. 8 contains the

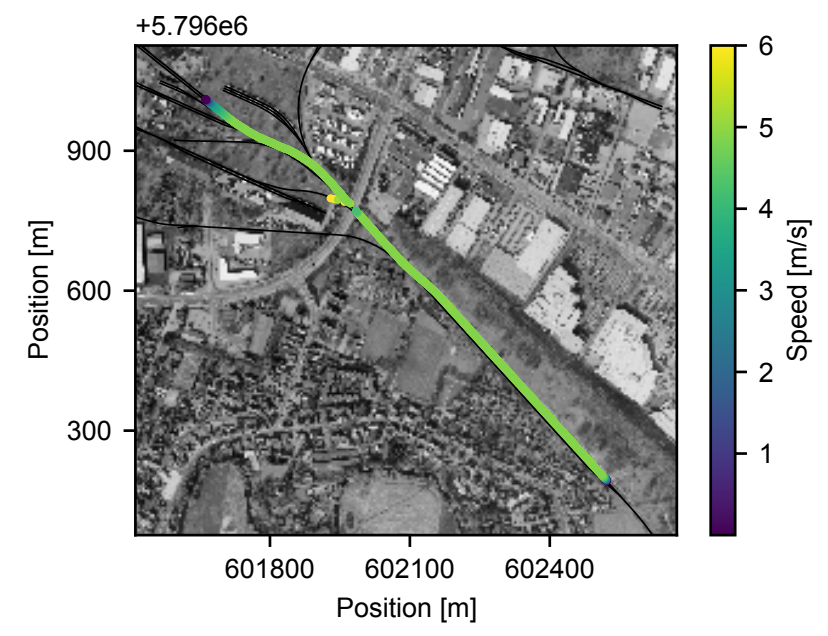

Fig. 6. The Javad GNSS data of a single journey as a scatter plot. Aerial photograph: Stadt Braunschweig, Abteilung Geoinformation.

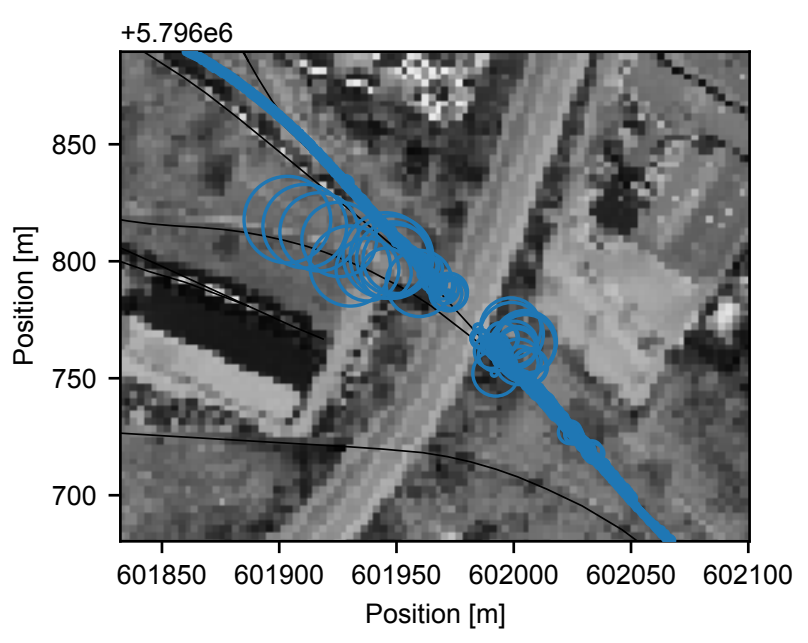

Fig. 7. The Javad GNSS data of an entire session in the vicinity of a bridge above the rails. The circles illustrate the uncertainty as provided by the receiver. Aerial photograph: Stadt Braunschweig, Abteilung Geoinformation.

distances as measured by the radar and odometer, which are consistent with one another. The integrated IMU acceleration deviates only mildly, which shows the bias stability of the Xsens device. The deviation of the optical sensor is due to a calibration error, which has been purposely left uncorrected and can be addressed by the reader in various ways. A similar mis-calibration of the optical sensor can be seen in the speed measurements in Fig. 9. These errors are examples of the practically relevant sensor characteristics that can be explored using the data set.

The basic processing steps which have to be applied to the raw data so that they conform to [10] are time synchronization, transformation into common reference frames, and the assignment of unified data labels. Details of these steps are described below. 


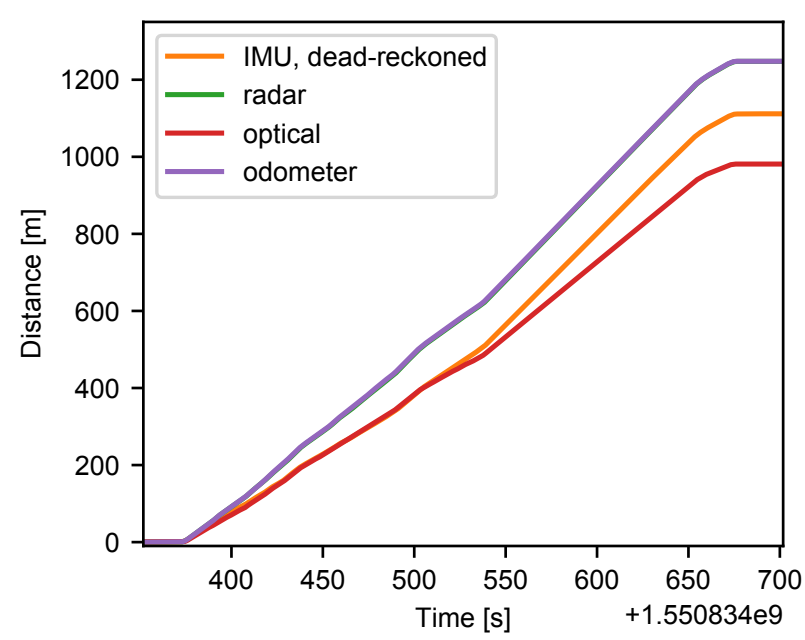

Fig. 8. Distance measurements by several sensors. Included are the distances obtained by integrating the longitudinal acceleration of the Xsens IMU, and measurements of the radar, optical sensor, and odometer. The radar and odometer can be seen as references here. The optical sensor deviates due to an intentionally uncorrected scale error.

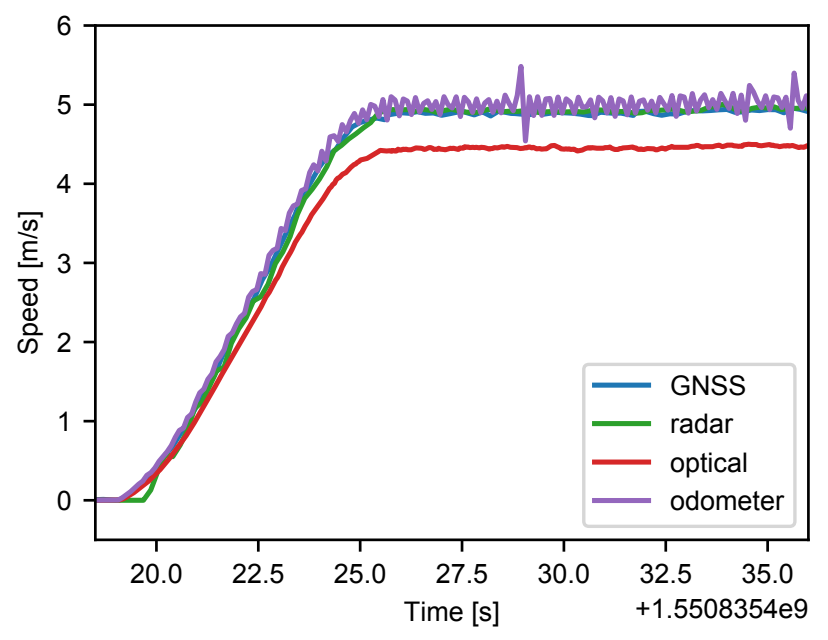

Fig. 9. Speed measurements by several sensors. Included are the Javad GNSS, radar, optical sensor, and odometer. The GNSS, radar, and odometer provide similar impressions. However, the GNSS is subject to outages under the bridge and the odometer can be affected by wheel slip.

1) Time Synchronization: Time synchronization incorporates the transformation of all sensor time stamps to Unix time, the synchronization of the RailDriVE data with the iNat-M200/STN data, and the limitation of all data to a common time window. A small remaining deviation between the RailDriVE and iNat-M200/STN time stamps (after conversion to Unix time) appeared because the RailDriVE was not synchronized perfectly with a global time reference. The offset was computed by cross-correlating IMU data and subsequently used for correction.

2) Transformation to Common Reference Frames: This processing step involves the transformation of the raw data from its sensor specific frames to a common vehicle, track or navigation frame respectively, according to [10]. The vehicle frame is chosen to be a right-handed Cartesian coordinate system located at the center of the front axle, with zero height at the level of the railheads, and the x-axis pointing to the front of the vehicle. Furthermore, the origin of the track frame is defined to be at the initial position of the vehicle, with positive counting into the direction of the vehicle's initial forwards alignment. The global position of the vehicle is specified in the navigation frame which is defined by geographic coordinates according to the World Geodetic System 1984 (WGS84).

As the GNSS receivers already provide their PVT output with reference to WGS84 no further transformations have to be applied to their data. The distance outputs of the odometer and the two speed sensors are transformed to the track frame by subtracting the initial offset. Last, the IMU data is aligned to the vehicle frame by applying appropriate rotation matrices. Lever-arm effects are not addressed in the preparation of the processed data. All required information to perform the above steps, e.g., the mounting positions of the sensors, are provided within the data set.

3) Data Formatting and Storage: The last processing steps applied to the raw data are preparations for them to be as easily usable as possible and for being stored in a commaseparated values (CSV) file. This includes the handling of sensor outages, the standardization of the units being used, the assignment of standardized data labels, and the storage of the data in CSV files.

Some of the raw data contain invalid data being already marked as invalid by the data collecting sensor, e.g. during GNSS outages or very slow driving. All data already being marked as invalid are replaced with NaNs. Furthermore, all measured quantities are converted to a standardized set of data labels and units as defined in [10]. An excerpt of exemplary definitions for IMU data is shown in Tab. I. This

TABLE I

EXCERPT OF FORMAT DEFINITIONS FOR IMU DATA ACCORDING TO [10]

\begin{tabular}{llll}
\hline Data & Label & Unit & Frame \\
\hline Time stamp (Unix) & TimeUnix_s & $\mathrm{s}$ & Unix Time \\
Acceleration (x-axis) & AccX_mss & $\mathrm{m} / \mathrm{s}^{2}$ & vehicle \\
Turn rate (x-axis) & TurnRateX_degs & $\%$ & \\
& $\ldots$ & & \\
& & & \\
\end{tabular}

conversion is a very important step in the processing of the raw data because this standardization allows the processed data for being easily interchangeable in the user's application with other processed data conforming to [10]. Finally, the processed data is stored in separate CSV files. To save storage space, NaNs are saved as empty fields.

\section{Reference Data}

Besides the raw and processed data, also reference data are provided conforming to the guidelines of [10]. This gives users the opportunity to evaluate and compare their results. 
In general it is difficult to provide an absolute positioning reference for railway positioning data. This is because it requires additional equipment, e.g., a total station or a reference GNSS receiver, along the track to gather an absolute positioning reference with the needed accuracy in the range of a few centimeters. In many cases it is not feasible to cover the entire test area with such additional equipment. Therefore, instead of an absolute reference, two different positioning solutions are provided here. These can be used as a relative reference for other positioning solutions. Furthermore, a precise track-map of the driven path is provided. The various reference data are explained below.

\section{1) Loosely Coupled GNSS/IMU Positioning Solution:} There are two positioning solutions available, which have been calculated by loosely coupling the GNSS/IMU data of the RailDriVE or the iNat-M200/STN, respectively. The Matlab implementation of the used extended Kalman filter (EKF) is fully included in the _scripts-folder in the data set. The EKF estimates the two dimensional position (latitude and longitude) of the vehicle in the navigation frame, its driven distance since the starting point, its velocity over ground, and its heading. The longitudinal vehicle acceleration and the yaw rate serve as input variables, whereas the latitude, longitude and velocity data from the GNSS receiver are incorporated via measurement updates. Thus, the EKF provides estimates of the horizontal position (latitude and longitude) and a velocity vector of the vehicle.

This simple EKF sensor fusion is provided as a fully open and comprehensible example that reflects a common sensor fusion strategy in positioning applications. Although this solution is not fully exploiting the potential of the fusion of GNSS/IMU data, it can be a good starting point for comparison with more sophisticated sensor fusion approaches.

2) Tightly Coupled GNSS/IMU Positioning Solution of iNat-M200/STN: The iNat-M200/STN provides a tightly coupled GNSS/IMU data fusion solution. It calculates the three-dimensional position, velocity and attitude of the vehicle. This solution can be considered more accurate than the one of the EKF above. The datasheet specifies a circular error probable (CEP) of $\pm 2 \mathrm{~m}$ for normal INS/GNSS operation and $\pm 0.4 \mathrm{~m}$ for INS/DGNSS operation. During the test drive the iNat-M200/STN's GNSS was available $97.8 \%$ of the time in which $97.7 \%$ of all GNSS solutions could be enhanced with a satellite based augmentation system (SBAS).

A disadvantage of the iNat-M200/STN reference is that the implementation and the underlying assumptions of the used filter are unknown.

3) Precise Track-map: In order to facilitate mapsupported positioning, a segment of the railway network data is included. The map data contains separate two-dimensional polylines for each track. Connections between tracks can be found from shared starting or end points.

\section{E. Usage}

In the _scripts-folder (c.f. Fig. 5) various Matlab scripts are included. They serve on the one hand documentation purposes and on the other hand they can be used as a starting point for working with the provided data.

From the script processRawData.m, which has been used to process the raw data, it becomes comprehensible how exactly the raw data have been modified to yield the processed data (c. f. Section III-C). If the provided processed data are not suitable for the application intended by a user, the script can also be used as a starting point for own modifications. Additionally, the script plotProcessedData.m allows to plot some of the processed data to quickly get an impression of the data at hand.

Also contained is the implementation of the EKF used to calculate the reference positioning solution presented in Section III-D.1. It can be started with the script calcEkfFusionData.m. It demonstrates how the processed data may be used and how the resulting positioning solution may be stored in accordance to [10]. Besides that, it can also be seen how the unified structure of the data set helps to easily change the input data of an algorithm without having to adapt the algorithm. The original input data can be easily overloaded. Hence, it is possible to calculate a new positioning solution from the combination of the RailDriVE's GNSS and the iNat-M200/STN's IMU or vice versa. Additionally, the script plotEkfFusionData.m allows to plot some of the positioning results.

\section{RESEARCH DIRECTIONS}

As a starting point for researchers, a number of positioning references are listed. Good references for information on GNSS and IMU, the core of most recent positioning approaches, are [11], [12]. The most relevant tools for combining the heterogeneous data with information about the vehicle motion are from the field of statistical sensor fusion and described in [13], [14]. Recent approaches for GNSS-based rail vehicle positioning are surveyed in [1], [15]. An early patent for GPS-supported positioning in the rail domain is [16]. Different map-supported approaches for positioning include [17]-[21]. Approaches without mapsupport include [7], [22], [23]. Approaches with sensors beyond this data set include [24]-[26].

The data set reflects some of the real world imperfections that are often encountered, also beyond positioning. It can be used to investigate aspects of synchronization, with related sensor information recorded at various rates by two completely separate systems. Furthermore, the individual error characteristics of the sensors can be explored and quantified in order to devise more realistic sensor models. Intelligent averaging or decimation schemes can be investigated, e.g., to improve the IMU data for use at lower rates. Bias, scale and other calibration errors can be explored, e.g. with the mis-calibrated Correvit speed data. The raw IMU data can be used to investigate automated alignment with the vehicle coordinate frame, an important aspect for the use of portable sensor systems. Further areas of interest include the systematic comparison of positioning (reference) data, which can be present at different rates and can be computed from different GNSS receivers with unknown antenna placement. 
Finally, mapping approaches [7], [23] can be investigated using the data.

The data set has been recorded using mostly highperformance or specialized hardware. It is planned to also include low-cost sensors with sub-optimal antenna placement in future measurement campaigns, e.g., to address aspects of portable sensor systems. Future experiments with novel sensor concepts, further cameras, and lidar are conceivable. The latter two can be used to bridge a technological gap between the rail and automotive domain and facilitate research on environment perception, an important topic beyond positioning.

\section{CONCLUding REMARKS}

A rich and openly available data set for rail positioning experiments, including raw and pre-processed data as well as reference and example solutions, has been presented in this article. The measurement set-up and structure of the data set have been presented in detail, so as to facilitate its use with limited efforts. It has not been our intention to provide an entirely polished data set but a solid basis for further discussions, research work, and student theses. Therefore, prospective research directions have been discussed. We hope that further ideas emerge from the use of the data.

The paper and data set are results of the authors' collaboration within the LRT initiative, a group of universities and research institutes that work on unifying and sharing data for the advance of rail vehicle positioning research and technology. This is the first data set published within the LRT initiative. Further measurement campaigns and open data sets are planned. We hope that this step towards more open research paradigms can inspire others and help advance the overall rail research.

\section{ACKNOWLEDGMENT}

The authors thank the partners of the LRT initiative for joint development of the guidelines used to prepare the data set, and the Geodetic Measurement Systems and Sensors group at TU Darmstadt for providing the IMU/GNSS sensor platform iNat-M200/STN.

\section{REFERENCES}

[1] J. Marais, J. Beugin, and M. Berbineau, "A Survey of GNSS-Based Research and Developments for the European Railway Signaling," IEEE Transactions on Intelligent Transportation Systems, vol. 18, no. 10, pp. 2602-2618, Oct. 2017.

[2] A. Geiger, P. Lenz, C. Stiller, and R. Urtasun, "Vision meets robotics: The KITTI dataset," The International Journal of Robotics Research, vol. 32, no. 11, pp. 1231-1237, Sep. 2013.

[3] H. Winter and M. Roth, "RailDriVE February 2019 - Data Set for Rail Vehicle Positioning Experiments," TUdatalib, 2020. [Online]. Available: https://doi.org/10.25534/tudatalib-166.2

[4] The LRT website. [Online]. Available: https://lrt-initiative.org

[5] K. Lüddecke and C. Rahmig, "Evaluating multiple GNSS data in a multi-hypothesis based map-matching algorithm for train positioning," in Proc. IEEE Intelligent Vehicles Symposium (IV), Jun. 2011, pp. 1037-1042.

[6] R. Schenkendorf, J. C. Groos, and L. Johannes, "Strengthening the Rail Mode of Transport by Condition Based Preventive Maintenance," IFAC-PapersOnLine, vol. 48, no. 21, pp. 964-969, Jan. 2015.
[7] S. Shankar, M. Roth, L. A. Schubert, and J. A. Verstegen, "Automatic Mapping of Center Line of Railway Tracks using Global Navigation Satellite System, Inertial Measurement Unit and Laser Scanner," Remote Sensing, vol. 12, no. 3, p. 411, Jan. 2020.

[8] M. Quigley, K. Conley, B. Gerkey, J. Faust, T. Foote, J. Leibs, R. Wheeler, and A. Ng, "ROS: an open-source Robot Operating System," in ICRA Workshop on Open Source Software, vol. 3, 2009.

[9] T. Schreiner and S. Dietiker, "Corrail1000 - An Optical Sensor for Contactless and Slip Free Speed Measurement for Rail Vehicles," in Proc. Scientific-Expert Conference on Railways (RAILCON'18), 2018.

[10] "LRT data sharing principles," LRT Initiative, May 2020, version: 1.2. [Online]. Available: https://lrt-initiative.org/documents

[11] P. J. Teunissen and O. Montenbruck, Eds., Springer Handbook of Global Navigation Satellite Systems. Cham, Switzerland: Springer International Publishing, 2017.

[12] P. D. Groves, Principles of GNSS, inertial, and multisensor integrated navigation systems, 2nd ed. Boston: Artech House, 2013.

[13] F. Gustafsson, Statistical Sensor Fusion. Lund, Sweden: Studentlitteratur, 2010.

[14] S. Särkkä, Bayesian filtering and smoothing. Cambridge, UK: Cambridge University Press, 2013.

[15] J. Otegui, A. Bahillo, I. Lopetegi, and L. E. Díez, "A Survey of Train Positioning Solutions," IEEE Sensors Journal, vol. 17, no. 20, pp. 6788-6797, Oct. 2017.

[16] R. D. Burns, S. Elliott-Bryan, and D. B. Turner, "Rail vehicle positioning system," US Patent US5 129605A, Jul., 1992.

[17] S. S. Saab, "A map matching approach for train positioning. I. Development and analysis," IEEE Transactions on Vehicular Technology, vol. 49, no. 2, pp. 467-475, Mar. 2000

[18] K. Gerlach and C. Rahmig, "Multi-hypothesis based map-matching algorithm for precise train positioning," in Proc. 12th International Conference on Information Fusion (FUSION), Jul. 2009, pp. 13631369.

[19] M. Lauer and D. Stein, "A Train Localization Algorithm for Train Protection Systems of the Future," IEEE Transactions on Intelligent Transportation Systems, vol. 16, no. 2, pp. 970-979, Apr. 2015.

[20] O. Heirich, "Bayesian Train Localization with Particle Filter, Loosely Coupled GNSS, IMU, and a Track Map," Journal of Sensors, vol. 2016, p. Article ID 2672640, 2016.

[21] M. Roth, B. Baasch, P. Havrila, and J. Groos, "Map-Supported Positioning Enables In-Service Condition Monitoring of Railway Tracks," in Proc. 21st International Conference on Information Fusion (FUSION), Jul. 2018, pp. 2346-2353.

[22] H. Winter, V. Willert, and J. Adamy, "Increasing Accuracy in Train Localization Exploiting Track-Geometry Constraints," in Proc. 21st International Conference on Intelligent Transportation Systems (ITSC), Nov. 2018, pp. 1572-1579.

[23] H. Winter, S. Luthardt, V. Willert, and J. Adamy, "Generating Compact Geometric Track-Maps for Train Positioning Applications," in Proc. IEEE Intelligent Vehicles Symposium (IV), Jun. 2019, pp. 1027-1032.

[24] S. Hensel, C. Hasberg, and C. Stiller, "Probabilistic Rail Vehicle Localization With Eddy Current Sensors in Topological Maps," IEEE Transactions on Intelligent Transportation Systems, vol. 12, no. 4, pp. 1525-1536, Dec. 2011.

[25] O. Heirich, B. Siebler, and E. Hedberg, "Study of Train-Side Passive Magnetic Measurements with Applications to Train Localization," Journal of Sensors, vol. 2017, p. Article ID 8073982, 2017.

[26] M. Spindler and M. Lauer, "High Accuracy Estimation of Velocity and Position for Railway Vehicles using Ferromagnetic Inhomogeneities," in Proc. 21st International Conference on Intelligent Transportation Systems (ITSC), Nov. 2018, pp. 1202-1207. 